\title{
On Asymptotic Solutions of the Functional Difference Equations Associated with Some Nonlinear Difference Equations
}

\author{
By \\ Sen-ichiro TANAKA*

\section{Introduction}

Let

$$
y(x+1)=g(x, y)
$$

be a system of $m$ nonlinear difference equations for the vector-valued function $y(x)=\left(y_{1}(x), y_{2}(x), \ldots, y_{m}(x)\right)$ of a complex variable $x . g(x, y)$ is an analytic function of $m+1$ complex variables $(x, y)$ defined in the region $X_{0} \times Y_{0}$, where

$$
\begin{aligned}
& X_{0}:|x|>R, \\
& Y_{0}:\|y\|<r \quad\|y\|=\max _{j}\left|y_{j}\right|,
\end{aligned}
$$

$R, r$ being positive constants. When we consider the expansion

$$
g(x, y)=g_{0}(x)+A(x) y+\sum_{|p| \geqq 2} g_{p}(x) y^{p},
$$

the case in which $g_{0}(x) \neq \equiv 0$ was discussed in [1], and the case in which $g_{0}(x) \equiv 0, A(x) \neq 0$ was discussed in [2]. We shall deal with some cases in which $g_{0}(x) \equiv 0$ and $A(x) \equiv 0$.

In Part II we shall discuss about a system of nonlinear difference equations of the form:

$$
y_{i}(x+1)=\prod_{j=1}^{m} y_{j}^{\lambda_{i j}} f_{i}(x, y) \quad(i=1,2, \ldots, m)
$$

Received February 6, 1970.

Communicated by M. Urabe.

* Toyama University. 
where $\lambda_{i j}$ 's are nonnegative integers such that

$$
\sum_{j=1}^{m} \lambda_{i j} \geqq 2 \quad(i=1,2, \cdots, m)
$$

and $f_{i}(x, y)$ are analytic functions of $x$ and $y$ in the region $X_{0} \times Y_{0}$ such that $f_{i}(\infty, 0) \neq 0(i=1,2, \cdots, m)$. Under the further assumption that each eigenvalue $\lambda_{i}$ of the $m \times m$ matrix $\Lambda=\left(\lambda_{i j}\right)$ is absolutely greater than one it may be shown that without loss of generality we may assume $f_{i}(\infty, 0)=1$.

In $\S 6$ we shall prove the existence of a transformation of the form:

$$
y_{i}=u_{i}\left(1+\sum_{|k| \geqq 1} p_{k_{0} k_{1} \cdots k_{m}}^{(i)} x^{-k_{0}} u_{1}^{k_{1}} u_{2}^{k_{2}} \cdots u_{m}^{k_{m}}\right) \quad(i=1,2, \ldots, m)
$$

by which the equation (0.3) are transformed into the most simple equations

$$
u_{i}(x+1)=\prod_{j=1}^{m} u_{j}^{\lambda_{i j}} \quad(i=1,2, \ldots, m)
$$

so that we can conclude that the equation (0.3) has a formal solution of the form (0.4) in which $u_{i}$ is substituted by any solution $u_{i}(x)$ of the equation (0.5).

On the basis of this situation we find it effective to regard the solution (0.4) as a function $y_{i}(x, u)$ of $m+1$ independent variables $x, u_{1}$, $u_{2}, \ldots, u_{m}$ satisfying the following equations:

$$
\begin{aligned}
& y_{i}(x\left.+1, \prod_{j=1}^{m} u_{j}^{\lambda_{1 j}}, \prod_{j=1}^{m} u_{j}^{\lambda_{2 j}}, \ldots, \prod_{j=1}^{m} u_{j}^{\lambda_{m j}}\right) \\
&=\prod_{j=1}^{m} y_{j}^{\lambda_{i j}}(x, u) f_{i}(x, y(x, u)) \quad(i=1,2, \ldots, m)
\end{aligned}
$$

which we shall call a system of functional difference equations associated with the original system of difference equations (0.3). In $\S 7$ we shall give a detailed discussion on this matter.

In $\S 8$ and $\S 9$ we shall prove the existence and the uniqueness of an analytic solution of $(0.6)$ of the form 


$$
\begin{array}{r}
y_{i}(x, u)=u_{i}\left(1+\sum_{|k|=1}^{N} p_{k_{0} k_{1} \ldots k_{m}}^{(i)} x^{-k_{0}} u_{1}^{k_{1}} u_{2}^{k_{2}} \cdots u_{m}^{k_{m}}+z_{i N}(x, u)\right) \\
(i=1,2, \ldots, m)
\end{array}
$$

such that the inequality

$$
|| z_{N} \| \leqq M\left\{|x|^{-(N+1)}+\|u\|^{N+1}\right\}
$$

holds with some arbitrary constant $M$ in a certain region of $(x, u)$ space. In $\S 9$ we shall show the existence of an asymptotic analytic solution of (0.6).

In preparation for the discussions in Part II, we shall deal with a following particular case of equation (0.3) in Part I:

$$
y_{i}(x+1)=y_{i}^{\lambda_{i}} f_{i}(x, y) \quad(i=1,2, \ldots, m),
$$

where $\lambda_{i}$ 's are integers $\geqq 2$.

Equations of the form $(0.8)$ are important in themselves since they appear in the study of some important nonlinear difference equations. For example, consider a nonlinear difference equation of the form:

$$
z(x+1)=a_{0}(x)+a_{1}(x) z(x)+\cdots+a_{m}(x) z^{m}(x) \quad(m \geqq 2),
$$

where $a_{j}(x)(j=0,1, \ldots, m)$ are analytic functions of $x$ in the region $|x|>R$ such that $a_{m}(\infty) \neq 0$. By the transformation $z=1 / y$, equation (0.9) is transformed to the following equation of the form (0.8):

$$
y(x+1)=y^{m} f(x, y)
$$

where

$$
f(x, y)=\left(a_{m}(x)+a_{m-1}(x) y+\cdots \cdots+a_{0}(x) y^{m}\right)^{-1} .
$$

Any solution of (0.9) that approaches to $\infty$ as $x$ tends to $\infty$ is then obtained from a solution of (0.10) that approaches to 0 as $x$ tends to $\infty$.

In conclusion the author expresses his hearty thanks to Professor HUKUHARA, Professor SIBAGAKI and Professor URABE who had through the preparation of this paper incessant interest on our problem and gave many criticisms and improvements. 


\section{Part 耳. On the System of Nonlinear Difference Equations of the Form $y_{i}(x+\mathbb{1})=y_{i}^{\lambda_{i}} f_{i}(x, y) \quad(i=\mathbb{1}, 2, \ldots, m)$}

1. Formal solutions. We consider the system of nonlinear difference equations of the form

$$
y_{i}(x+1)=y_{i}^{\lambda_{i}} f_{i}\left(x, y_{1}, y_{2}, \ldots, y_{m}\right) \quad(i=1,2, \ldots, m)
$$

where $\lambda_{i}$ 's are integers greater than one and $f_{i}$ 's are analytic functions of $x$ and $y=\left(y_{1}, y_{2}, \ldots, y_{m}\right)$ which are defined in the domain $X_{0} \times Y_{0}$ $=\{x|| x \mid>R\} \times\{y \mid\|y\|<r\}\left(\|y\|=\max _{i}\left|y_{i}\right|\right)$ and satisfy the conditions

$$
f_{i}(\infty, 0,0, \ldots, 0)=\mu_{i} \neq 0 \quad(i=1,2, \ldots, m) .
$$

We remark that we may assume $\mu_{i}=1(i=1,2, \ldots, m)$ without loss of generality. Indeed, setting $\xi_{i}=\mu_{i}^{1 /\left(\lambda_{i}-1\right)}$ and introducing the unknown $z_{i}=\xi_{i} y_{i}$, we have for $z_{i}$ the equations

$$
z_{i}(x+1)=z_{i}^{\lambda_{i}} g_{i}\left(x, z_{1}, z_{2}, \ldots, z_{m}\right),
$$

where $g_{i}\left(x, z_{1}, z_{2}, \ldots, z_{m}\right)=\mu_{i}^{-1} f_{i}\left(x, \xi_{1}^{-1} z_{1}, \xi_{2}^{-1} z_{2}, \ldots, \xi_{m}^{-1} z_{m}\right)$ for which it holds

$$
g_{i}(\infty, 0,0, \ldots, 0)=1 \quad(i=1,2, \ldots, m) .
$$

Owing to the assumptions just made we have the expansions

$$
f_{i}\left(x, y_{1}, y_{2}, \cdots, y_{m}\right)=1+\sum_{|k| \geqq 1} a_{k_{0} k_{1} \cdots k_{m}}^{(i)} x^{-k_{0}} y_{1}^{k_{1}} y_{2}^{k_{2}} \cdots y_{m}^{k_{m}}
$$

where $|k|=k_{0}+k_{1}+\cdots+k_{m}$.

In finding a formal solution of the system of equations (1.1) we will show that a transformation of the type

$$
y_{i}=u_{i}\left(1+\sum_{|k| \geqq 1} p_{k_{0} k_{1} \cdots k_{m}}^{(i)} x^{-k_{0}} u_{1}^{k_{1}} u_{2}^{k_{2}} \cdots u_{m}^{k_{m}}\right)
$$

is effective, the coefficients $p_{k_{0} k_{1} \ldots k_{m}}^{(i)}$ being suitably chosen.

First, we consider the transformation of the first step

$$
y_{i}=u_{i}\left(1+q_{0}^{(i)} x^{-1}+\sum_{j=1}^{m} q_{j}^{(i)} u_{j}\right)
$$


which has the inverse transformation

$$
u_{i}=y_{i}\left(1-q_{0}^{(i)} x^{-1}-\sum_{j=1}^{m} q_{j}^{(i)} y_{j}+\cdots\right)
$$

where $\ldots$ represents the terms which are of higher degree than one in $x^{-1}$ and $y_{j}$. Setting $x+1$ for $x$ in (1.5) and using (1.1) and (1.4) it is immediately shown that the new unknown $u_{i}$ satisfies the following equation

$$
\begin{aligned}
u_{i}(x+1)= & u_{i}^{\lambda_{i}}\left(1+q_{0}^{(i)} x^{-1}+\sum_{j=1}^{m} q_{j}^{(i)} u_{j}\right)^{\lambda_{i}} \\
& \times\left(1+\sum_{|k|=1} a_{k_{0} k_{1} \cdots k_{m}}^{(i)} x^{-k_{0}} u_{1}^{k_{1}} u_{2}^{k_{2}} \cdots u_{m}^{k_{m}}+\cdots\right) \\
& \times\left(1-q_{0}^{(i)} x^{-1}+\cdots\right),
\end{aligned}
$$

i.e.,

$$
\begin{aligned}
u_{i}(x+1)= & u_{i}^{\lambda_{i}}\left\{1+\left(\left(\lambda_{i}-1\right) q_{0}^{(i)}+a_{10 \ldots 0}^{(i)}\right) x^{-1}\right. \\
& \left.+\sum_{j=1}^{m}\left(\lambda_{i} q_{j}^{(i)}+a_{00 \ldots 10 \ldots 0}^{(i)}\right) u_{j}+\cdots\right\}
\end{aligned}
$$

where $\ldots$ represents higher terms in $x^{-1}$ and $u_{j}$. Now by the assumption $\lambda_{i} \geqq 2$ we can choose the coefficients $q_{0}^{(i)}, q_{j}^{(i)}(j=1,2, \ldots, m)$ so that the coefficients of $x^{-1}$ and $u_{j}$ in the right-hand side of (1.6) may all vanish, in other words, equation (1.6) may be of the form

$$
u_{i}(x+1)=u_{i}^{\lambda_{2}}\left(1+\sum_{|k| \geqq 2} b_{k_{0} k_{1} \cdots k_{m}}^{(i)} x^{-k_{0}} u_{1}^{k_{1}} u_{2}^{k_{2}} \cdots u_{m}^{k_{m}}\right) .
$$

This is of the form similar to (1.1) but the terms of degree one in $x^{-1}$ and $u_{j}$ are lacking in the right-hand expansion. Now, to this equation we perform the transformation of the second step

$$
u_{i}=v_{i}\left(1+\sum_{|k|=2} r_{k_{0} k_{1} \cdots k_{m}}^{(i)} x^{-k_{0}} v_{1}^{k_{1}} v_{2}^{k_{2}} \ldots v_{m}^{k_{m}}\right),
$$

which has the inverse transformation

$$
v_{i}=u_{i}\left(1-\sum_{|k|=2} r_{k_{0} k_{1} \cdots k_{m}}^{(i)} x^{-k_{0}} u_{1}^{k_{1}} u_{2}^{k_{2}} \cdots u_{m}^{k_{m}}+\cdots\right) .
$$

Then, it can be shown as before that the new unknown $v_{i}$ satisfies the 
equation of the form

$$
\begin{aligned}
v_{i}(x+1)= & v_{i}^{\lambda_{2}}\left\{1+\left(\left(\lambda_{i}-1\right) r_{200 \ldots 0}^{(i)}+b_{200 \ldots 0}^{(i)}\right) x^{-2}\right. \\
& \left.+\sum_{|k|=2}^{\prime}\left(\lambda_{i} r_{k_{0} k_{1} \ldots k_{m}}^{(i)}+b_{k_{0} k_{1} \ldots k_{m}}^{(i)}\right) x^{-k_{0}} v_{1}^{k_{1}} v_{2}^{k_{2}} \ldots v_{m}^{k_{m}}+\cdots\right\}
\end{aligned}
$$

Here it is observed that the expansion \{\} has no terms of degree one $x^{-1}$ and $v_{i}$, and $\cdots$ means terms of degree higher than two in $x^{-1}$ and $v_{i}$. Now by $\lambda_{i} \geqq 2$ we can choose the coefficients $r_{k_{0} k_{1} \ldots k_{m}}^{(i)}$ for $|k|=2$ so that the coefficients of $x^{-2}$ and $x^{-k_{0}} v_{1}^{k_{1}} v_{2}^{k_{2}} \ldots v_{m}^{k_{m}}$ for $|k|=2$ may all vanish, in other words, the resulted equation for the unknown $v$ may be of the form

$$
v_{i}(x+1)=v_{i}^{\lambda_{i}}\left(1+\sum_{|k| \geqq 3} c_{k_{0} k_{1} \ldots k_{m}}^{(i)} x^{-k_{0}} v_{1}^{k_{1}} v_{2}^{k_{2}} \ldots v_{m}^{k_{m}}\right) .
$$

It has a form similar to (1.1) but the terms of degree one and two in $x^{-1}$ and $v$ 's are lacking in the right-hand expansion.

Repeating similar processes we have after $N$ steps $N$ transformations

$$
\begin{aligned}
& y_{i}=u_{i}\left(1+q_{0}^{(i)} x^{-1}+\sum_{j=1}^{m} q_{j}^{(i)} u_{j}\right) \\
& u_{i}=v_{i}\left(1+\sum_{|k|=2} r_{k_{0} k_{1} \cdots k_{m}}^{(i)} x^{-k_{0}} v_{1}^{k_{1}} v_{2}^{k_{2}} \cdots v_{m}^{k_{m}}\right) \\
& \quad \ldots \ldots \ldots \ldots \\
& u_{i, N-1}=u_{i, N}\left(1+\sum_{|k|=N} t_{k_{0} k_{1} \cdots k_{m}}^{(i)} x^{-k_{0}} u_{1, N}^{k_{1}} u_{2, N}^{k_{2}} \cdots u_{m, N}^{k_{m}}\right) .
\end{aligned}
$$

Here $u_{i}, v_{i}$ means, respectively, $u_{i, 1}$ and $u_{i, 2}$. As composite transformation from $\left\{y_{i}\right\}$ to $\left\{u_{i, N}\right\}$ we have

$$
y_{i}=u_{i, N}\left(1+\sum_{|k| \geqq 1} p_{k_{0} k_{1} \ldots k_{m, N}}^{(i)} x^{-k_{0}} u_{1, N}^{k_{1}} u_{2, N}^{k_{2}} \cdots u_{m, N}^{k_{m}}\right)
$$

and the equation satisfied by $u_{i N}$ has the form

$$
u_{i, N}(x+1)=u_{i, N}^{\lambda_{2}}\left\{1+\sum_{|k| \geqq N+1} b_{k_{0} k_{1} \cdots k_{m, N}}^{(i)} x^{-k_{0}} u_{1, N}^{k_{1}} u_{2, N}^{k_{2}} \cdots u_{m, N}^{k_{m}}\right\}
$$

It is observed from the nature of the process that in all transformations (1.7) the coefficient of $x^{-k_{0}} u_{1}^{k_{1}} u_{2}^{k_{2}} \cdots u_{m}^{k_{m}}$ preserves the value $p_{k_{0} k_{1} \ldots k_{m},|k|}^{(i)}$ for $N=|k|,|k|+1,|k|+2, \ldots \ldots$. Hence we may define the coefficients in (1.3) by setting 


$$
p_{k_{0} k_{1} \ldots k_{m}}^{(i)}=p_{k_{0} k_{1} \ldots k_{m},|k|}^{(i)}
$$

Thus we have defined the transformation (1.3) from $\left\{y_{i}\right\}$ to $\left\{u_{i}\right\}$. Now, two transformations (1.3) and (1.7) give a relation between $\left\{u_{i}\right\}$ and $\left\{u_{i, N}\right\}$ defined by

$$
\begin{aligned}
& u_{i}\left(1+\sum_{|k| \geqq 1} p_{k_{0} k_{1} \cdots k_{m}}^{(i)} x^{-k_{0}} u_{1}^{k_{1}} u_{2}^{k_{2}} \cdots u_{m}^{k_{m}}\right) \\
& \quad=u_{i, N}\left(1+\sum_{|k| \geqq 1} p_{k_{0} k_{1} \cdots k_{m}, N}^{(i)} x^{-k_{0}} u_{1, N}^{k_{1}} u_{2, N}^{k_{2}} \cdots u_{m, N}^{k_{m}}\right)
\end{aligned}
$$

which can be solved formally in the form

$$
u_{i}=u_{i, N}\left(1+\sum_{|k| \geqq 1} \bar{p}_{k_{0} k_{1} \cdots k_{m, N}}^{(i)} x^{-k_{0}} u_{1, N}^{k_{1}} u_{2, N}^{k_{2}} \cdots u_{m, N}^{k_{m}}\right),
$$

and as its inverse we have

$$
u_{i, N}=u_{i}\left(1+\sum_{|k| \geqq 1} \overline{\bar{p}}_{k_{0} k_{1} \cdots k_{m}, N}^{(i)} x^{-k_{0}} u_{1}^{k_{1}} u_{2}^{k_{2}} \cdots u_{m}^{k_{m}}\right)
$$

By the definition (1.9) we have in (1.10) and (1.11) the relations

$$
\bar{p}_{k_{0} k_{1} \cdots k_{m, N}}^{(i)}=\overline{\bar{p}}_{k_{0} k_{1} \cdots k_{m, N}}^{(i)}=0 \quad(1 \leqq|k| \leqq N),
$$

so that we have

$$
u_{i}=u_{i, N}\left(1+\sum_{|k| \geqq N+1} \bar{p}_{k_{0} k_{1} \ldots k_{m, N}}^{(i)} x^{-k_{0}} u_{1, N}^{k_{1}} u_{2, N}^{k_{2}} \cdots u_{m, N}^{k_{m}}\right)
$$

and

$$
u_{i, N}=u_{i}\left(1+\sum_{|k| \geqq N+1} \overline{\bar{p}}_{k_{0} k_{1} \cdots k_{m, N}}^{(i)} x^{-k_{0}} u_{1}^{k_{1}} u_{2}^{k_{2}} \cdots u_{m}^{k_{m}}\right)
$$

When these expressions are substituted into the equation (1.8) we find equation satisfied by $\left\{u_{i}\right\}$ are of the form:

$$
u_{i}(x+1)=u_{i}^{\lambda_{2}}\left(1+\sum_{|k| \geqq N+1} c_{k_{0} k_{1} \cdots k_{m}, N}^{(i)} x^{-k_{0}} u_{1}^{k_{1}} u_{2}^{k_{2}} \cdots u_{m}^{k_{m}}\right)
$$

Here $N$ has been an arbitrary integer, so that the equation satisfied by $\left\{u_{i}\right\}$ is really

$$
u_{i}(x+1)=u_{i}^{\lambda_{i}} .
$$


Thus we have proved that the equation (1.1) is reduced to (1.12) when the transformation given by (1.3) is performed on it. Consequently we can conclude that the equation (1.1) has a formal solution of the form

$$
y_{i}(x, u)=u_{i}\left(1+\sum_{|k| \geqq 1} p_{k_{0} k_{1} \ldots k_{m}}^{(i)} x^{-k_{0}} u_{1}^{k_{1}} u_{2}^{k_{2}} \cdots u_{m}^{k_{m}}\right),
$$

in which $u_{i}$ is substituted by a solution $u_{i}(x)$ of the equation (1.12).

2. Assceiated functional difference equations. Making use of the formal solution (1.13) of the equation (1.1) we shall conveniently deal with a solution of (1.1) of the form

$$
y_{i}(x)=y_{i}(x, u(x)) \quad(i=1,2, \ldots, m)
$$

depending on the general solution $u(x)=\left(u_{1}(x), u_{2}(x), \ldots, u_{m}(x)\right)$ of the system of equations: $u(x+1)=u^{\lambda}(x)$, i.e.,

$$
u_{i}(x+1)=u_{i}^{\lambda_{i}}(x) \quad(i=1,2, \ldots, m) .
$$

The general solution $u(x)$ of (2.2) is

$$
u_{i}(x)=\exp \left(\pi_{i}(x) \lambda_{i}^{x}\right)
$$

containing an arbitrary periodic function $\pi(x)=\left(\pi_{1}(x), \pi_{2}(x), \ldots, \pi_{m}(x)\right)$ with period 1 , so that the function (2.1) corresponding to (2.3) is a general solution of (1.1).

Now substitute (2.1) and (2.2) into (1.1), then we have

$$
y_{i}\left(x+1, u^{\lambda}\right)=y_{i}^{\lambda^{2}}(x, u) f_{i}[x, y(x, u)] \quad(i=1,2, \ldots, m) .
$$

This equality holds formally in $x$ and $u$, because $y=y(x, u)$ is a transformation reducing (1.1) to (1.12) formally in $x$ and $u$. Let us write (2.4) briefly as

$$
y_{i}\left(x+1, u^{\lambda}\right)=y_{i}^{\lambda^{2}} f_{i}(x, y) \quad(i=1,2, \ldots, m)
$$

and call this equation an associated functional difference equation of (1.1).

Clearly $y(x, u)$ given by (1.13) is a formal solution of (2.5). In order to prove the asymptoticity of this formal solution, let us put 


$$
y_{i}(x, u)=u_{i}\left(P_{i N}(x, u)+z_{i N}(x, u)\right),
$$

where

$$
P_{i N}(x, u)=1+\sum_{|k|=1}^{N} p_{k_{0} k_{1} \ldots k_{m}}^{(i)} x^{-k_{0}} u_{1}^{k_{1}} u_{2}^{k_{2}} \cdots u_{m}^{k_{m}} \quad(i=1,2, \ldots, m),
$$

thus introducing new unknown function $z_{N}(x, u)$.

Substituting them in (2.5), the equation for $z_{N}$ becomes

$$
\begin{aligned}
& z_{i N}\left(x+1, u^{\lambda}\right) \\
& \quad=\left(P_{i N}(x, u)+z_{i N}(x, u)\right)^{\lambda_{1}} f_{i}\left(x, u\left(P_{N}+z_{N}\right)\right)-P_{i N}\left(x+1, u^{\lambda}\right),
\end{aligned}
$$

where for simplicity we use the notation $u\left(P_{N}+z_{N}\right)$ for the vector $\left\{u_{1}\left(P_{1 N}+z_{1 N}\right), u_{2}\left(P_{2 N}+z_{2 N}\right), \ldots, u_{m}\left(P_{m N}+z_{m N}\right)\right\}$. Let $\varphi_{i N}(x, u, z)$ and $c_{i N}(x, u)$ be defined by

$$
\begin{aligned}
\varphi_{i N}(x, u, z) & =\left(P_{i N}+z_{i N}\right)^{\lambda_{2}} f_{i}\left(x, u\left(P_{N}+z_{N}\right)\right)-P_{i{ }_{i}}^{\lambda_{i}} f_{i}\left(x, u P_{N}\right), \\
c_{i N}(x, u) & =P_{i N}^{\lambda_{i}} f_{i}\left(x, u P_{N}\right)-P_{i N}\left(x+1, u^{\lambda}\right),
\end{aligned}
$$

then we get

$$
z_{i N}\left(x+1, u^{\lambda}\right)=\varphi_{i N}(x, u, z)+c_{i N}(x, u) \quad(i=1,2, \ldots, m) .
$$

Writing $z_{i N}, \varphi_{i N}, c_{i N}$, respectively, as $z_{i}, \varphi_{i}, c_{i}$ in (2.6) for simplicity, we have

$$
z_{i}\left(x+1, u^{\lambda}\right)=\varphi_{i}(x, u, z)+c_{i}(x, u) \quad(i=1,2, \ldots, m) .
$$

Owing to the assumption made on the functions $f_{i}$ in (1.1), and to the fact that $P_{i N}$ in (2.1) are polynomials in $x^{-1}$ and $u_{i}$, we see that there exist positive constants $R_{2}, r_{2}$ such that $c_{i}(x, u)$ are defined and analytic in

$$
|x|>R_{2}, \quad\|u\|<r_{2}
$$

Referring to the fact that (1.3) is a formal solution of (1.1) we can conclude that there exists a positive constant $L$ such that the inequality

$$
\|c(x, u)\| \leqq L\left\{|x|^{-(N+1)}+\|u\|^{N+1}\right\}
$$


holds in the region (2.8). Choosing positive constants $R_{3}, r_{3}$ suitably we see that $\varphi_{i}(x, u, z)$ are analytic in the region

$$
|x|>R_{3}, \quad\|\mid u\|<r_{3}, \quad\|z\|<r_{3}
$$

and moreover we have

$$
\varphi_{i}(x, u, 0)=0 \quad(i=1,2, \ldots, m) .
$$

Therefore we may write $\varphi(x, u, z)$ in the form:

$$
\varphi(x, u, z)=B(x, u) z+\phi(x, u, z) .
$$

Here $B(x, u)$ is an $m$ by $m$ matrix $B(x, u)=\left(b_{i k}(x, u)\right)$, whose elements are analytic functions in the region $|x|>R_{3},\|u\|<r_{3}$ such that

$$
\begin{aligned}
b_{i i}(x, u) & =\frac{\partial}{\partial z_{i}} \varphi_{i}(x, u, 0) \\
& =\lambda_{i} P_{i}^{\lambda_{i}-1} f_{i}(x, u P)+u_{i} P_{i}^{\lambda_{i}} \frac{\partial}{\partial y_{i}} f_{i}(x, u P), \\
b_{i k}(x, u) & =u_{k} P_{i}^{\lambda_{i}} \frac{\partial}{\partial y_{k}} f_{i}(x, u P) \quad(i \neq k),
\end{aligned}
$$

so that it holds

$$
B(\infty, 0)=\operatorname{diag}\left(\lambda_{1}, \lambda_{2}, \ldots, \lambda_{m}\right)
$$

$\psi(x, u, z)$ is an analytic function in the region (2.10), and there exists a positive quantity $K$ such that the inequality

$$
\|\phi(x, u, z)\| \leqq K\|z\|^{2}
$$

holds in (2.10). Thus (2.6) may be written as

$$
z_{i}\left(x+1, u^{\lambda}\right)=c_{i}(x, u)+\sum_{k=1}^{m} b_{i k}(x, u) z_{k}+\phi_{i}(x, u, z)
$$

3. Existence theorem. In this section, we shall prove that the functional difference equation (2.11) has an analytic solution such that

$$
\|z(x, u)\| \leqq M\left\{|x|^{-(N+1)}+\|u\|^{N+1}\right\}
$$


in a certain region $\Gamma \times U$ such as

$$
\begin{aligned}
& \Gamma:|x|>\rho_{1}, \quad|\arg x|<\delta, \\
& U:\|u\|<\rho_{2}(<1),
\end{aligned}
$$

where $M$ and $\delta$ are arbitrary but fixed constants such that $M>L$ and $\delta<\frac{\pi}{2}$, and the constants $\rho_{1}$ and $\rho_{2}$ will be determined in the course of proof. We shall employ the Fixed-Point Theorem to prove this.

Let $F$ be a family of functions $z(x, u)$ that are analytic and satisfying the inequality (3.1) for $x$ and $u$ in the region $\Gamma \times U . F$ is a convex set, because if $f(x, u)$ and $g(x, u)$ belong to $F$ the inequality

$$
\begin{aligned}
& \|\lambda f(x, u)+(1-\lambda) g(x, u)\| \\
& \quad \leqq \lambda \mid f(x, u)\|+(1-\lambda)\| g(x, u) \| \\
& \quad \leqq M\left\{|x|^{-(N+1)}+\|u\|^{N+1}\right\}
\end{aligned}
$$

holds in $\Gamma \times U$ for any $\lambda$ such as $0<\lambda<1$. $F$ is a closed set, because if $\left\{z_{i}(x, u)\right\}$ is a sequence of functions belonging to $F$, and converging uniformly in any compact set in $\Gamma \times U$, then the limiting function $z(x, u)$ satisfies (3.1) and is analytic in $\Gamma \times U$, which means that $z(x, u)$ belongs to $F$.

Now, referring to the equation (2.11) consider the mapping $T$ defined as

$$
\begin{aligned}
& \bar{z}(x, u)=T(z(x, u)) \\
&=\Lambda^{-1}\left\{z\left(x+1, u^{\lambda}\right)-c(x, u)-H(x, u) z-\psi(x, u, z)\right\}
\end{aligned}
$$

where $H(x, u)=B(x, u)-\Lambda, \Lambda=\operatorname{diag}\left(\lambda_{1}, \lambda_{2}, \ldots, \lambda_{m}\right)$. We may choose positive constants $L, B, K, R^{\prime}, r^{\prime}(<1)$ and $r^{\prime \prime}(<1)$ such that the inequalities

$$
\begin{aligned}
& \| c(x, u) ! \leqq L\left\{|x|^{-(N+1)}+\|u\|^{N+1}\right\} \\
& \|H(x, u)\| \leqq B\left\{|x|^{-1}+\|u\|\right\}, \\
& \|\psi(x, u, z)\| \leqq K\|z\|^{2}
\end{aligned}
$$


hold in the region $|x|>R^{\prime},\|u\|<r^{\prime}$ and $\|z\|<r^{\prime \prime}$. We notice that if $z(x, u) \in F$, then $z\left(x+1, u^{\lambda}\right) \in F$.

Now, $\left\|\Lambda^{-1}\right\|=\max _{i} \lambda_{i}^{-1} \leqq \frac{1}{2}$ and $M>L$, we have from (3.2)

$$
\begin{aligned}
& \mid i \bar{z}(x, u) \| \\
& \leqq \frac{1}{2}\left\{\left\|z\left(x+1, u^{\lambda}\right)\right\|+\left|{ }_{1} c(x, u)\|+\| H(x, u)\|\| z\|+\| \phi(x, u, z)\right|_{i}\right\} \\
& \leqq \frac{M}{2}\left\{|x|^{-(N+1)}+\|u\|^{N+1}\right\} \\
& \quad \times\left[1+\frac{L}{M}+B\left(|x|^{-1}+\|u\|\right)+K M\left(|x|^{-(N+1)}+\|\left. u\right|^{N+1}\right)\right],
\end{aligned}
$$

so that choosing $\rho_{1}$ sufficiently large and $\rho_{2}<1$ sufficiently small we have

$$
\|\bar{z}(x, u)\| \leqq M\left\{|x|^{-(N+1)}+\|u\|^{N+1}\right\}
$$

in the region $\Gamma \times U$. Thus the mapped function $\bar{z}(x, u)$ which is clearly analytic in $\Gamma \times U$ belongs also to $F$.

If $\left\{z_{n}(x, u)\right\}$ is a sequence of functions that belong to $F$ and converges uniformly in any compact set in $\Gamma \times U$, then the sequence $\left\{\bar{z}_{n}(x, u)\right\}$ of the mapped functions clearly converges uniformly to a function $\bar{z}(x, u)$ in $\Gamma \times U$ which is the image of the limiting function $z(x, u)$.

Lastly we notice that the family of the mapped functions forms a normal family in $\Gamma \times U$. This is clear from the fact that $\bar{z}_{n}(x, u)$ are analytic and equibounded by $\left\|\bar{z}_{n}(x, u)\right\| \leqq M\left\{|x|^{-(N+1)}+\|u\|^{N+1}\right\}$.

Hence all the necessary assumptions of the Fixed-Point Theorem were shown to be satisfied in our case, so that we have the following existence theorem.

Theorem 1. Let $f_{i}(x, y)$ be analytic in the region $X_{0} \times Y_{0}$ such as

$$
\begin{aligned}
& X_{0}:|x|>R, \\
& Y_{0}:\|y\|<r \quad \text { where } \quad\|y\|=\max _{i}\left|y_{i}\right|
\end{aligned}
$$

and let us consider the functional difference equation 


$$
y_{i}\left(x+1, u^{\lambda}\right)=y_{i}^{\lambda_{i}} f_{i}(x, y),
$$

where $y=y(x, u)$ is a function of independent variables $x$ and $u=\left(u_{1}, u_{2}\right.$, $\left.\ldots, u_{m}\right)$. Then in accordance with the following formal solution

$$
y_{i}=u_{i}\left\{1+\sum_{|k| \geqq 1} p_{k_{0} k_{1} \ldots k_{m}}^{(i)} x^{-k_{0}} u_{1}^{k_{1}} u_{2}^{k_{2}} \cdots u_{m}^{k_{m}}\right\}
$$

of (2.5) there exists correspondingly to an arbitrary but fixed quantity $M$ (which is greater than the quantity $L$ introduced in (2.9)) an analytic solution

$$
y_{i}=u_{i}\left(1+\sum_{|k|=1}^{N} p_{k_{0} k_{1} \ldots k_{m}}^{(i)} x^{-k_{0}} u^{k_{1}} u^{k_{2}} \cdots u^{k_{m}}+z_{i N}\right)
$$

such that the inequality

$$
\left\|z_{N}\right\| \leqq M\left\{|x|^{-(N+1)}+\|u\|^{N+1}\right\}
$$

holds in a cerlain region $\Gamma_{N} \times U_{N}$ which is defined for a sufficiently large quantity $\rho_{1 N}$ and a sufficiently small quantity $\rho_{2 N}<1$ as

$$
\begin{aligned}
& \Gamma_{N}:|x|>\rho_{1 N}, \quad|\arg x|<\delta\left(<\frac{\pi}{2}\right), \\
& U_{N}:\|u\|<\rho_{2 N} .
\end{aligned}
$$

4. Uniqueness theorem. We shall consider the uniqueness of the solution of the functional difference equation

$$
z\left(x+1, u^{\lambda}\right)=c(x, u)+B(x, u) z(x, u)+\psi(x, u, z) .
$$

We have proved that it has an analytic and bounded solution in a region $\Gamma \times U$. We shall show that such a solution is unique.

Supposing two solutions $z_{1}(x, u)$ and $z_{2}(x, u)$ we set

$$
v(x, u)=z_{2}(x, u)-z_{1}(x, u) \text {. }
$$

Then $v$ satisfies the equation

$$
v\left(x+1, u^{\lambda}\right)=B(x, u) v+\psi\left(x, u, z_{1}+v\right)-\psi\left(x, u, z_{1}\right) .
$$

Expanding the right-hand side in power of $v$, we can write the above 
equation in the form

$$
v\left(x+1, u^{\lambda}\right)=A\left(x, u, z_{1}\right) v+\Psi\left(x, u, z_{1}, v\right)
$$

where $\Psi$ denotes the part containing terms of degrees higher than one in $v$. By assumption $A\left(x, u, z_{1}\right)$ is analytic in the region $X_{1} \times U_{1} \times Z_{1}$ and $\Psi\left(x, u, z_{1}, v\right)$ is analytic in the region $X_{1} \times U_{1} \times Z_{1} \times V_{1}$ where

$$
\begin{aligned}
& D_{1}:|x|>R_{1}, \quad|\arg x|<\delta, \\
& U_{1}:\|u\|<r_{1}, \\
& Z_{1}:\left\|z_{1}\right\|<\bar{r}_{1}, \\
& V_{1}:\|v\|<\bar{r}_{1}
\end{aligned}
$$

for some suitable quantities $R_{1}, r_{1}, \bar{r}_{1}, \overline{\bar{r}}_{1}$, and it holds $A(\infty, 0,0)=\Lambda$. Set

$$
H\left(x, u, z_{1}\right)=A\left(x, u, z_{1}\right)-\Lambda \text {. }
$$

Since $\lambda_{0}=\min _{i} \lambda_{i} \geqq 2$, we can select a number $\rho$ such that $\lambda_{0}>\rho>1$. Then for the positive quantity $\lambda_{0}-\rho$ we choose $R_{2}>0$ sufficiently large, $r_{2}<0$ sufficiently small so that in the region $V=D_{2} \times U_{2} \times Z_{2} \times V_{2}$ such as

$$
\begin{aligned}
& D_{2}:|x|>R_{2}, \quad|\arg x|<\delta, \\
& U_{2}:\|u\|<r_{2}, \\
& Z_{2}:\left\|z_{1}\right\|<M\left(R_{2}^{-(N+1)}+r_{2}^{N+1}\right), \\
& V_{2}:\|v\|<2 M\left(R_{2}^{-(N+1)}+r_{2}^{N+1}\right)
\end{aligned}
$$

the inequality

$$
\left\|H\left(x, u, z_{1}\right) v+\Psi\left(x, u, z_{1}, v\right)\right\| \leqq\left(\lambda_{0}-\rho\right)\|v\|
$$

holds. It follows that

$$
\|\Lambda v\|-\|A(x, u, z) v+\Psi(x, u, z, v)\| \leqq\left(\lambda_{0}-\rho\right)\|v\| .
$$

Since $\lambda_{0}\|v\| \leqq\|\Lambda v\|$, we have ultimately

$$
\left\|A\left(x, u, z_{1}\right)+\Psi\left(x, u, z_{1}, v\right)\right\| \geqq \rho\|v\|,
$$

so that by (4.2) we have 


$$
\left\|v\left(x+1, u^{\lambda}\right)\right\| \geq \rho\|v(x, u)\|
$$

Since $v(x, u)$ is bounded in the region $D_{2} \times U_{2}$, putting

$$
S=\sup _{D_{2} \times U_{2}}\|v(x, u)\|
$$

we get $S \geqq \rho S$ where $\rho>1$. So we must have $S=0$. Hence $z_{1}(x, u)$ $=z_{2}(x, u)$ in $D_{2} \times U_{2}$. Since the solution is analytic $\Gamma \times U$, such a solution is unique in $\Gamma \times U$. Thus we get the following theorem.

Theorem 2. Under the conditions of Theorem 1 the solution stated in it is unique.

5. Existence of an asymptotic solution. We have proved that for the equation

$$
y_{i}\left(x+1, u^{\lambda}\right)=y_{i}^{\lambda_{i}} f_{i}(x, y) \quad(i=1,2, \ldots, m)
$$

there exists an analytic solution

$$
y_{i}=u_{i}\left(P_{i N}(x, u)+z_{i N}(x, u)\right),
$$

where

$$
P_{i N}(x, u)=1+\sum_{|k|=1}^{N} p_{k_{0} k_{1} \cdots k_{m}}^{(i)} x^{-k_{0}} u_{1}^{k_{1}} u_{2}^{k_{2}} \cdots u_{m}^{k_{m}}
$$

such as $\left\|z_{N}\right\| \leqq M\left\{|x|^{-(N+1)}+\|u\|^{N+1}\right\}$, and that such a solution is unique in $\Gamma_{N} \times U_{N}$. The solution $y_{i}=y_{i}(x, u)$ might depend on $N$, so that temporarity let us denote it by $y_{i}[N]$. Then

$$
y_{i}[N]=u_{i}\left(P_{i N}(x, u)+z_{i N}(x, u)\right),
$$

where $\left\|z_{N}\right\| \leqq M\left\{|x|^{-(N+1)}+\|u\|^{N+1}\right\}$ in $\Gamma_{N} \times U_{N}$. Now this can be rewritten as

$$
y_{i}[N]=u_{i}\left(P_{i, N-1}(x, u)+v_{i, N-1}(x, u)\right)
$$

where

$$
v_{i, N-1}(x, u)=\sum_{|k|=N} p_{k_{0} k_{1} \cdots k_{m}}^{(i)} x^{-k_{0}} u_{1}^{k_{1}} u_{2}^{k_{2}} \cdots u_{m}^{k_{m}}+z_{i N}(x, u)
$$


Since $\left\|z_{N}\right\| \leqq M\left\{|x|^{-(N+1)}+\|u\|^{N+1}\right\}$ in $\Gamma_{N} \times U_{N}$, it follows that

$$
\left\|v_{N-1}\right\| \leqq M_{N-1}^{\prime}\left\{|x|^{-N}+\left\|u_{\eta}\right\|^{N}\right\}
$$

where $M_{N-1}^{\prime}$ is some positive quantity. On the other hand, according to uniqueness theorem the solution such as

$$
y_{i}[N-1]=u_{i}\left(P_{i, N-1}(x, u)+z_{i, N-1}(x, u)\right)
$$

where $\left\|z_{N-1}\right\| \leqq M_{N-1}\left\{|x|^{-N}+\|u\|^{N}\right\}$ in $\Gamma_{N-1} \times U_{N-1}$ is unique, so that it must hold

$$
y_{i}[N]=y_{i}[N-1]
$$

and consequently

$$
\begin{aligned}
z_{i, N-1}(x, u)=\sum_{|k|=N} p_{k_{0} k_{1} \ldots k_{m}}^{(i)} x^{-k_{0}} u_{1}^{k_{1}} u_{2}^{k_{2}} \cdots u_{m}^{k_{m}}+z_{i N}(x, u) \\
\quad \text { in } \quad\left(\Gamma_{N-1} \cap \Gamma_{N}\right) \times\left(U_{N-1} \cap U_{N}\right) .
\end{aligned}
$$

Therefore we can conclude that there exists an analytic solution which does not depend on $N$ in some region of the form previously denoted by $\Gamma \times U$, having the property that it holds

$$
\begin{aligned}
& y_{i}(x, u)=u_{i}\left(P_{i N}(x, u)+z_{i N}(x, u)\right), \\
& \left\|z_{N}(x, u)\right\| \leqq M_{N}\left\{|x|^{-(N+1)}+\|u\|^{N+1}\right\} \quad \text { in } \quad \Gamma \times U,
\end{aligned}
$$

which means that we have asymptotically

$$
y_{i}(x, u) \sim u_{i}\left(1+\sum_{|k| \geqq 1} p_{k_{0} k_{1} \cdots k_{m}}^{(i)} x^{-k_{0}} u_{1}^{k_{1}} u_{2}^{k_{2}} \cdots u_{m}^{k_{m}}\right)
$$

in $\Gamma \times U$. Thus we have

Theorem 3. Under the conditions of Theorem 1 there exists an analytic asymptotic solution of the equation (2.5) such as

$$
y_{i}(x, u) \sim u_{i}\left(1+\sum_{|k| \geqq 1} p_{k_{0} k_{1} \ldots k_{m}}^{(i)} x^{-k_{0}} u_{1}^{k_{1}} u_{2}^{k_{2}} \cdots u_{m}^{k_{m}}\right)
$$

in the region $\Gamma \times U$. 
Part II. On the System of Nonlinear Difference Equations of the

$$
\text { Form } \quad y_{i}(x+1)=\prod_{j=1}^{m} y_{j}^{\lambda_{i} y} f_{i}(x, y) \quad(i=1,2, \ldots, m)
$$

6. Formal solutions. We consider the system of nonlinear difference equations of the form:

$$
y_{i}(x+1)=\prod_{j=1}^{m} y_{j}^{\lambda_{i j}} f_{i}(x, y) \quad(i=1,2, \ldots, m)
$$

where $\lambda_{i j}$ 's are nonnegative integers such that $\sum_{j=1}^{m} \lambda_{i j} \geqq 2(i=1,2, \ldots, m)$. We assume that $f_{i}(x, y)=f_{i}\left(x, y_{1}, y_{2}, \ldots, y_{m}\right)$ are analytic functions of $x$ and $y=\left(y_{1}, y_{2}, \ldots, y_{m}\right)$ defined in the region $X_{0} \times Y_{0}=\{x|| x \mid>R\}$ $\times\{y \mid\|y\|<r\}\left(\|y\|=\max _{i}\left|y_{i}\right|\right)$. Clearly functions $f_{i}(x, y)$ then can be expanded in the following form:

$$
f_{i}(x, y)=a_{00 \ldots 0}^{(i)}+\sum_{|k| \geqq 1} a_{k_{0} k_{1} \ldots k_{m}}^{(i)} x^{-k_{0}} y_{1}^{k_{1}} y_{2}^{k_{2}} \ldots y_{m}^{k_{m}}
$$

where $|k|$ means $k_{0}+k_{1}+\cdots+k_{m}$. We assume that $f_{i}(\infty, 0)=a_{00 \ldots 0}^{(i)} \equiv \mu_{i}$ $\neq 0(i=1,2, \ldots, m)$. Furthermore we assume that each eigenvalue $\lambda_{i}$ of the $m$ by $m$ matrix $\Lambda=\left(\lambda_{i j}\right)$ is absolutely greater than one.

We may assume $f_{i}(\infty, 0) \equiv \mu_{i}=1(i=1,2, \ldots, m)$ without loss of generality. Indeed, putting

$$
z_{i}=\xi_{i} y_{i} \quad(i=1,2, \ldots, m)
$$

with undetermined constants $\xi_{i}$ and substituting (6.3) in (6.1), we get the following system of difference equations for $z=\left(z_{1}, z_{2}, \ldots, z_{m}\right)$ :

$$
\prod_{j=1}^{m} \xi_{j}^{\lambda_{j}{ }_{i j}} z_{i}(x+1)=\xi_{i} \mu_{i} \prod_{j=1}^{m} z_{j}^{\lambda_{i j}} g_{i}(x, z) \quad(i=1,2, \ldots, m)
$$

where

$$
\begin{aligned}
g_{i}(x, z) & =g_{i}\left(x, z_{1}, z_{2}, \ldots, z_{m}\right) \\
& =\mu_{i}^{-1} f_{i}\left(x, \xi_{1}^{-1} z_{1}, \xi_{2}^{-1} z_{2}, \ldots, \xi_{m}^{-1} z_{m}\right)
\end{aligned}
$$

By our assumption one is not an eigenvalue of the matrix $\Lambda=\left(\lambda_{i j}\right)$ so that 
the constants $\xi_{i}(i=1,2, \ldots, m)$ can be so chosen that the equalities

$$
\prod_{j=1}^{m} \xi_{j}^{\lambda_{i j}}=\xi_{i} \mu_{i} \quad(i=1,2, \ldots, m)
$$

hold. By such choice of the constant $\xi=\left(\xi_{1}, \xi_{2}, \ldots, \xi_{m}\right)$, (6.4) has the required form:

$$
z_{i}(x+1)=\prod_{j=1}^{m} z_{j}^{\lambda_{i j}} g_{i}(x, z) \quad(i=1,2, \ldots, m)
$$

$g_{i}(x, z)$ are analytic functions in $X_{0} \times Z_{0}$ where

$$
\begin{aligned}
& X_{0}:|x|>R, \\
& Z_{0}:\|z\|<r^{\prime} \quad\left(r^{\prime}=r \min _{i}\left|\xi_{i}\right|\right),
\end{aligned}
$$

and they can be expanded in the same form as (6.2) but with $a_{00 \ldots 0}^{(i)}=1$.

In this section, we shall show that we can find a formal solution of (6.1) by means of a transformation of the form:

$$
y_{i}=u_{i}\left(1+\sum_{|k| \geqq 1} p_{k_{0} k_{1} \cdots k_{m}}^{(i)} x^{-k_{0}} u_{1}^{k_{1}} u_{2}^{k_{2}} \cdots u_{m}^{k_{m}}\right)
$$

Adopting the same idea used in Part I, we shall decompose the transformation (6.7) in a series of steps.

First we take $u_{0}(x)=y(x)$. Then we consider the transformation from $y(x)$ to $u_{1}(x)$ defined by the relation of the form

$$
y_{i}=u_{i 1}\left(1+q_{0}^{(i)} x^{-1}+\sum_{n=1}^{m} q_{n}^{(i)} u_{n_{1}}\right)
$$

which has the inverse transformation

$$
u_{i 1}=y_{i}\left(1-q_{0}^{(i)} x^{-1}-\sum_{n=1}^{m} q_{n}^{(i)} y_{n}+\cdots\right)
$$

where $\ldots$ represents terms which are of degree higher than one in $x^{-1}$, $y_{1}, y_{2}, \ldots, y_{m}$. Setting $x+1$ for $x$ in (6.9) and using (6.1) and (6.8), it is immediately shown that the new unknown $u_{1}$ satisfies the following equation 


$$
\begin{aligned}
u_{i 1}(x+1)= & \prod_{j=1}^{m} u_{j 1}^{\lambda_{i j}}\left(1+q_{0}^{(j)} x^{-1}+\sum_{n=1}^{m} q_{n}^{(j)} u_{n 1}\right)^{\lambda_{\iota \jmath}} \\
& \times\left(1+\sum_{|k| \geqq 1} a_{k_{0} k_{1} \cdots k_{m}}^{(i)} x^{-k_{0}} u_{11}^{k_{1}} u_{21}^{k_{2}} \cdots u_{m 1}^{k_{m}}\right) \\
& \times\left(1-q_{0}^{(i)} x^{-1}-\cdots\right) .
\end{aligned}
$$

Let $E$ be the unit matrix and $M=\left(\mu_{i j}\right)$ be the $m$ by $m$ matrix defined by $M=\Lambda-E$. Then (6.10) becomes

$$
\begin{aligned}
u_{i 1}(x+1)= & \prod_{j=1}^{m} u_{j 1}^{\lambda_{2 j}}\left\{1+\left(\sum_{j=1}^{m} \mu_{i j} q_{0}^{(j)}+a_{10 \ldots 0}^{(i)}\right) x^{-1}\right. \\
& \left.+\sum_{n=1}^{m}\left(\sum_{j=1}^{m} \lambda_{i j} q_{n}^{(j)}+a_{00 \ldots 010 \ldots 0}^{(i)}\right) u_{n 1}+\cdots\right\}
\end{aligned}
$$

By the assumption one and zero are not the eigenvalue of $\Lambda$, so that $\Lambda$ and $M$ are regular matrices. Hence we can determine the constants $q_{0}^{(i)}$ and $q_{n}^{(i)}$ appearing in (6.8) so that the coefficients of $x^{-1}$ and $u_{n 1}$ in the right-hand side of (6.11) may all vanish.

The next step which transforms $u_{1}(x)$ to $u_{2}(x)$ is similar. Inductively let us assume that we already have the system of difference equations for $u_{N-1}$ of the form:

$$
u_{i, N-1}(x+1)=\prod_{j=1}^{m} u_{j, N-1}^{\lambda_{i j}}\left(1+\sum_{|k| \geqq N} \beta_{k_{0} k_{1} \cdots k_{m}, N-1}^{(i)} x^{-k_{0}} u_{1, N-1}^{k_{1}} \cdots u_{m, N-1}^{k_{m}}\right) .
$$

We shall show that a transformation from $u_{N-1}$ to $u_{N}$ of the form

$$
u_{i, N-1}(x+1)=u_{i, N}\left(1+\sum_{|k|=N} \gamma_{k_{0} k_{1} \cdots k_{m}, N}^{(i)} x^{-k_{0}} u_{1, N}^{k_{1}} u_{2, N}^{k_{2}} \cdots u_{m, N}^{k_{m}}\right)
$$

can be determined in such a way that we may have the system of difference equations for $u_{N}$ of the form:

$$
u_{i, N}(x+1)=\prod_{j=1}^{m} u_{j, n}^{\lambda_{2 j}}\left(1+\sum_{|k| \geqq N+1} \beta_{k_{0} k_{1} \cdots k_{m, N}}^{(i)} x^{-k_{0}} u_{1, N}^{k_{1}} u_{2, N}^{k_{2}} \cdots u_{m, N}^{k_{m}}\right) .
$$

Now (6.13) has the inverse transformation

$$
u_{i, N}=u_{i, N-1}\left(1-\sum_{|k|=N} \gamma_{k_{0} k_{1} \cdots k_{m, N}}^{(i)} x^{-k_{0}} u_{1, N-1}^{k_{1}} \cdots u_{m, N-1}^{k_{m}}+\cdots\right)
$$


where $\ldots$ shows the terms which are of degree higher than $N$ in $x^{-1}$, $u_{1, N-1}, u_{2, N-1}, \ldots, u_{m, N-1}$. Setting $x+1$ for $x$ in (6.15), (6.12) and (6.13), it is immediately shown that the new unknown $u_{i, N}$ satisfy the following equations

$$
\begin{aligned}
u_{i N}(x+1)= & \prod_{j=1}^{m} u_{j N}^{\lambda_{i j}}\left(1+\sum_{|k|=N} \gamma_{k_{0} k_{1} \cdots k_{m}, N}^{(j)} x^{-k_{0}} u_{1 N}^{k_{1}} \cdots u_{m N}^{k_{m}}\right)^{\lambda_{i j}} \\
& \times\left(1+\sum_{|k|=N} \beta_{k_{0} k_{1} \cdots k_{m}, N-1}^{(i)} x^{-k_{0}} u_{1 N}^{k_{1}} \cdots u_{m N}^{k_{m}}+\cdots\right) \\
& \times\left(1-\gamma_{N 0 \ldots, N}^{(i)} x^{-N}+\cdots\right),
\end{aligned}
$$

i.e.,

$$
\begin{aligned}
u_{i N}(x+1)= & \prod_{j=1}^{m} u_{j N}^{\lambda_{i j}}\left\{1+\left(\sum_{j=1}^{m} \mu_{i j} \gamma_{N 0 \ldots 0, N}^{(j)}+\beta_{N 0 \ldots 0, N-1}^{(i)}\right) x^{-N}\right. \\
& +\sum_{\substack{k \mid=N N \\
k_{0} \neq N}}\left(\sum_{j=1}^{m} \lambda_{i j} \gamma_{k_{0} k_{1} \ldots k_{m, N}}^{(j)}+\beta_{k_{0} k_{1} \ldots k_{m}, N-1}^{(i)}\right) x^{-k_{0}} u_{1 N}^{k_{1}} \cdots u_{m N}^{k_{m}} \\
& +\cdots\} .
\end{aligned}
$$

Since the matrices $M=\left(\mu_{i j}\right)$ and $\Lambda=\left(\lambda_{i j}\right)$ are regular, we can determine the coefficients $\gamma_{k_{0} k_{1} \ldots k_{m}, N}^{(i)}$ so that in the right-hand side of (6.16) all the coefficients of the terms of degree $N$ in $x^{-1}, u_{1 N}, \ldots, u_{m N}$ may vanish, that is, (6.6) may be of the form (6.14).

Now, by the composition of the mappings

$$
\begin{aligned}
y_{i}(x)= & u_{i 0}(x), \\
u_{i 0}= & u_{i 1}\left(1+q_{0}^{(i)} x^{-1}+\sum_{n=1}^{m} q_{n}^{(i)} u_{n, 1}\right), \\
u_{i 1}= & u_{i 2}\left(1+\sum_{|k|=2} \gamma_{k_{0} k_{1} \ldots k_{m}, 2}^{(i)} x^{-k_{0}} u_{12}^{k_{1}} u_{22}^{k_{2}} \cdots u_{m 2}^{k_{m}}\right), \\
& \ldots \ldots \ldots \ldots \ldots \\
u_{i, N-1}= & u_{i, N}\left(1+\sum_{|k|=N} \gamma_{k_{0} k_{1} \ldots k_{m}, N}^{(i)} x^{-k_{0}} u_{1 N}^{k_{1}} u_{2 N}^{k_{2}} \cdots u_{m N}^{k_{m}}\right) \\
& (i=1,2, \ldots, m),
\end{aligned}
$$

we get the following transformation from $y$ to $u_{N}$ : 


$$
y_{i}=u_{i N}\left(1+\sum_{|k| \geqq 1} p_{k_{0} k_{1} \cdots k_{m} . N}^{(i)} x^{-k_{0}} u_{1 N}^{k_{1}} u_{2 N}^{k_{2}} \cdots u_{m N}^{k_{m}}\right) .
$$

It is observed from the nature of the process that in all transformations (6.17) the coefficient of $x^{-k_{0}} u_{1 N}^{k_{1}} u_{2 N}^{k_{2}} \cdots u_{m N}^{k_{m}}$ preserves the value $p_{k_{0} k_{1} \cdots k_{m},|k|}^{(i)}$ for $N=|k|,|k|+1,|k|+2, \ldots$. Hence we may define the coefficients in (6.7) by setting

$$
p_{k_{0} k_{1} \ldots k_{m}}^{(i)}=p_{k_{0} k_{1} \ldots k_{m},|k|}^{(i)}
$$

Thus we have defined the transformation (6.7) from $y$ to $u$. Now, the two transformations (6.7) and (6.17) give a relation between $u$ and $u_{N}$ defined as

$$
\begin{aligned}
& u_{i}\left(1+\sum_{|k| \geqq 1} p_{k_{0} k_{1} \cdots k_{m}}^{(i)} x^{-k_{0}} u_{1}^{k_{1}} u_{2}^{k_{2}} \cdots u_{m}^{k_{m}}\right) \\
& \quad=u_{i N}\left(1+\sum_{|k| \geqq 1} p_{k_{0} k_{1} \cdots k_{m}, N}^{(i)} x^{-k_{0}} u_{1 N}^{k_{1}} u_{2 N}^{k_{2}} \cdots u_{m N}^{k_{m}}\right)
\end{aligned}
$$

which can be solved formally in the form

$$
u_{i}=u_{i N}\left(1+\sum_{|k| \geqq 1} \bar{p}_{k_{0} k_{1} \cdots k_{m} N}^{(i)} x^{-k_{0}} u_{1 N}^{k_{1}} u_{2 N}^{k_{2}} \cdots u_{m N}^{k_{m}}\right)
$$

and as its inverse we have

$$
u_{i N}=u_{i}\left(1+\sum_{|k| \geqq 1} \overline{\bar{p}}_{k_{0} k_{1} \cdots k_{m}, N}^{(i)} x^{-k_{0}} u_{1}^{k_{1}} u_{2}^{k_{2}} \cdots u_{N}^{k_{m}}\right)
$$

By the definition (6.18) we have in (6.20) and (6.21) the relations

$$
\bar{p}_{k_{0} k_{1} \ldots k_{m}, N}^{(i)}=\overline{\bar{p}}_{k_{0} k_{1} \ldots k_{m}, N}^{(i)}=0
$$

for $k$ such as $1 \leqq|k| \leqq N$, so that we have

$$
u_{i}=u_{i N}\left(1+\sum_{|k| \geqq N+1} \bar{p}_{k_{0} k_{1} \cdots k_{m}, N}^{(i)} x^{-k_{0}} u_{1 N}^{k_{1}} u_{2 N}^{k_{2}} \cdots u_{m N}^{k_{m}}\right),
$$

and

$$
u_{i N}=u_{i}\left(1+\sum_{|k| \geqq N+1} \overline{\bar{p}}_{k_{0} k_{1} \cdots k_{m}, N}^{(i)} x^{-k_{0}} u_{1}^{k_{1}} u_{2}^{k_{2}} \cdots u_{m}^{k_{m}}\right)
$$

When these expressions are substituted into the equation (6.14) we find 
that the equations satisfied by $\left\{u_{i}\right\}$ are of the form

$$
u_{i}(x+1)=\prod_{j=1}^{m} u_{j}^{\lambda_{i} j_{j}}\left(1+\sum_{|k| \geqq N+1} c_{k_{0} k_{1} \cdots k_{m}, N}^{(i)} x^{-k_{0}} u_{1}^{k_{1}} u_{2}^{k_{2}} \cdots u_{m}^{k_{m}}\right) .
$$

Here $N$ has been an arbitrary integer, so that the equation satisfied by $\left\{u_{i}\right\}$ is really

$$
u_{i}(x+1)=\prod_{j=1}^{m} u_{j}^{\lambda_{i j}}
$$

Thus we have proved that the equation (6.1) is reduced to (6.25) when the transformation given by (6.7) is performed on it. Consequently we can conclude that the equation (6.1) has a formal solution of the form

$$
y_{i}(x, u)=u_{i}\left(1+\sum_{|k| \geqq 1} p_{k_{0} k_{1} \cdots k_{m}}^{(i)} x^{-k_{0}} u_{1}^{k_{1}} u_{2}^{k_{2}} \cdots u_{m}^{k_{m}}\right)
$$

in which $u_{i}$ is substituted by a solution $u_{i}(x)$ of the equation (6.25).

7. Associated functional difference equation. Making use the formal solution (6.7) of the equation (6.1) we shall conveniently deal with a solution of (6.1) of the form

$$
y_{i}(x, u(x)) \quad(i=1,2, \ldots, m)
$$

depending on the general solution $u(x)=\left(u_{1}(x), u_{2}(x), \ldots, u_{m}(x)\right)$ of the system of equations

$$
u_{i}(x+1)=\prod_{j=1}^{m} u_{j}(x)^{\lambda_{2 \jmath}} \quad(i=1,2, \ldots, m) .
$$

The general solution $u(x)$ of (7.2) may be found easily by putting $v_{i}(x)$ $=\log u_{i}(x) . \quad u(x)$ contains an arbitrary periodic function $\pi(x)=\left(\pi_{1}(x)\right.$, $\left.\pi_{2}(x), \ldots, \pi_{m}(x)\right)$ with period one, so that the function (7.1) corresponding to the general solution $u(x)$ of (7.2) is a general solution of (7.1).

Substitute (7.1) and (7.2) into (6.1), then we have

$$
\begin{aligned}
y\left(x+1, \pi u^{\Lambda}\right)=\prod_{j=1}^{m} y_{j}^{\lambda_{i j}}(x, u) f_{i}[x, y(x, u)] & \\
& (i=1,2, \ldots, m),
\end{aligned}
$$


where the left-hand side denotes the rather complicated quantity

$$
y_{i}\left(x+1, \prod_{j=1}^{m} u_{j}^{\lambda_{1 j}}, \prod_{j=1}^{m} u_{j}^{\lambda_{2 j}}, \ldots, \prod_{j=1}^{m} u_{j}^{\lambda_{m j}}\right) .
$$

Similarly to (2.4), equality (7.3) holds formally in $x$ and $u$ for $y(x, u)$ given by (6.7). In what follows, we shall call (7.3) an associated functional difference equation of (6.1).

Corresponding to the formal solution (6.7) of the associated functional difference equation (7.3), let us put

$$
\begin{gathered}
y_{i}(x, u)=u_{i} P^{(i)}(x, u), \\
y_{i}(x, u)=u_{i}\left(P_{i N}(x, u)+z_{i N}(x, u)\right)
\end{gathered}
$$

where

$$
P^{(i)}(x, u)=1+\sum_{|k| \geqq 1} p_{k_{0} k_{1} \ldots k_{m}}^{(i)} x^{-k_{0}} u_{1}^{k_{1}} u_{2}^{k_{2}} \cdots u_{m}^{k_{m}}
$$

$$
P_{i N}(x, u)=1+\sum_{|k|=1}^{N} p_{k_{0} k_{1} \cdots k_{m}}^{(i)} x^{-k_{0}} u_{1}^{k_{1}} u_{2}^{k_{2}} \cdots u_{m}^{k_{m}}
$$

Substituting (7.5) in (7.3) the equation for $z_{N}$ becomes

$$
\begin{aligned}
z_{i N} & \left(x+1, \pi u^{\Lambda}\right) \\
= & \prod_{j=1}^{m}\left(P_{j N}(x, u)+z_{j N}(x, u)\right)^{\lambda_{i j}} f_{i}\left(x, u\left(P_{N}+z_{N}\right)\right) \\
& \quad-P_{i N}\left(x+1, \pi u^{\Lambda}\right)
\end{aligned}
$$

where

$$
\begin{aligned}
& f_{i}\left(x, u\left(P_{N}+z_{N}\right)\right) \\
& \quad=f_{i}\left(x, u_{1}\left(P_{1 N}+z_{1 N}\right), u_{2}\left(P_{2 N}+z_{2 N}\right), \ldots, u_{m}\left(P_{m N}+z_{m N}\right)\right),
\end{aligned}
$$

and

$$
\begin{aligned}
& P_{i N}\left(x+1, \pi u^{\Lambda}\right) \\
& \quad \equiv P_{i N}\left(x+1, \prod_{j=1}^{m} u_{j}^{\lambda_{1 j}}, \prod_{j=1}^{m} u_{j}^{\lambda_{2 j}}, \ldots, \prod_{j=1}^{m} u_{j}^{\lambda_{m j}}\right) .
\end{aligned}
$$


Let $\varphi_{i N}(x, u, z)$ and $c_{i N}(x, u)$ be defined by

$$
\begin{gathered}
\varphi_{i N}(x, u, z)=\prod_{j=1}^{m}\left(P_{j N}+z_{j N}\right)^{\lambda_{\imath \jmath}} f_{i}\left(x, u\left(P_{N}+z_{N}\right)\right) \\
\quad-\prod_{j=1}^{m}\left(P_{j N}\right)^{\lambda_{\imath \jmath}} f_{i}\left(x, u P_{N}\right) \\
c_{i N}(x, u)=\prod_{j=1}^{m}\left(P_{j N}(x, u)\right)^{\lambda_{i j}} f_{i}\left(x, u P_{N}\right)-P_{i N}\left(x+1, \pi u^{\Lambda}\right) .
\end{gathered}
$$

Then we get

$$
z_{i N}\left(x+1, \pi u^{\Lambda}\right)=\varphi_{i N}(x, u, z)+c_{i N}(x, u) \quad(i=1,2, \ldots, m) .
$$

Writing for simplicity $z_{i N}, \varphi_{i N}, c_{i N}$, respectively, as $z_{i}, \varphi_{i}, c_{i}$ in this equation we shall study the following functional difference equation:

$$
z_{i}\left(x+1, \pi u^{\Lambda}\right)=\varphi_{i}(x, u, z)+c_{i}(x, u) \quad(i=1,2, \ldots, m) .
$$

Owing to the assumption on the functions $f_{i}$ in (7.8) and to the fact that $P_{i N}$ in (7.6) are polynomials in $x^{-1}$ and $u_{i}$, we see that there exist positive constants $R_{2}, r_{2}$ such that $c_{i}(x, u)$ defined by (7.8) are analytic in the region

$$
|x|>R_{2}, \quad\|u\|<r_{2} .
$$

Referring to the fact that (6.7) is a formal solution of (7.3), we get the formal equalities

$$
\prod_{j=1}^{m} u_{j}^{\lambda_{i j}} P^{(i)}\left(x+1, \pi u^{\Lambda}\right)=\prod_{j=1}^{m}\left(u_{j} P^{(j)}(x, u)\right)^{\lambda_{2 j}} f_{i}(x, u P(x, u))
$$

that is,

$$
\begin{array}{r}
\prod_{j=1}^{m}\left(P^{(j)}(x, u)\right)^{\lambda_{i j}} f_{i}(x, u P(x, u))-P^{(i)}\left(x+1, \pi u^{\Lambda}\right)=0 \\
(i=1,2, \ldots, m) .
\end{array}
$$

By (7.6), (7.11) and the definition (7.8) of $c_{i}(x, u)$, we get the estimation

$$
c_{i}(x, u)=\mathrm{O}\left(|x|^{-(N+1)}+\|u\|^{N+1}\right) \quad(i=1,2, \ldots, m) .
$$


Therefore we can conclude that there exists a positive constant $L$ such that

$$
\|c(x, u)\| \supseteq L\left\{|x|^{-(N+1)}+\|u\|^{N+1}\right\}
$$

holds in (7.10). Choosing positive constants $R_{3}, r_{3}$ suitably we see that $\varphi_{i}(x, u, z)$ are analytic in the region

$$
|x|>R_{3}, \quad\|u\|<r_{3}, \quad\|z\|<r_{3},
$$

and moreover we have $\varphi_{i}(x, u, 0)=0(i=1,2, \ldots, m)$. Therefore we may write $\varphi=\left(\varphi_{1}, \varphi_{2}, \ldots, \varphi_{m}\right)$ in the form

$$
\varphi(x, u, z)=B(x, u) z+\psi(x, u, z) .
$$

Here $B(x, u)$ is an $m$ by $m$ matrix $B(x, u)=\left(b_{i k}(x, u)\right)$ whose elements are analytic in $|x|>R_{3},\left\|_{1} u\right\|<r_{3}$ such that

$$
\begin{aligned}
b_{i k}(x, u)= & \frac{\lambda_{i k}}{P_{k N}} \prod_{j=1}^{m}\left(P_{j N}\right)^{\lambda_{i j}} f_{i}\left(x, u P_{N}\right) \\
& +u_{k} \prod_{j=1}^{m}\left(P_{j N}\right)^{\lambda_{i j}} \frac{\partial f_{i}}{\partial y_{k}}\left(x, u P_{N}\right) .
\end{aligned}
$$

Hence we have

$$
B(\infty, 0)=\left(\lambda_{i k}\right)-\Lambda
$$

$\psi(x, u, z)$ is an analytic function in (7.12), and there exists a positive quantity $K$ such that the inequality

$$
\|\psi(x, u, z)\| \leqq K\|z\|^{2}
$$

holds in (7.12). Therefore (7.9) may be written as

$$
\begin{array}{r}
z_{i}\left(x+1, \pi u^{\Lambda}\right)=c_{i}(x, u)+\sum_{j=1}^{m} b_{i j}(x, u) z_{j}+\phi_{i}(x, u, z) \\
(i=1,2, \cdots, m) .
\end{array}
$$

Let us put $\lambda_{0}=\min _{i}\left|\lambda_{i}\right|$. By assumption we have $\lambda_{0}>1$. For an arbitrary but fixed constant $\delta$ such as 


$$
\lambda_{0}-1>\delta>0
$$

there exists a regular matrix $S$ such that

$$
\Lambda_{J}=S^{-1} \Lambda S \quad\left(\Lambda=\left(\lambda_{i j}\right)\right)
$$

where $\Lambda_{J}$ is a Jordan's canonical form of $\Lambda$ :

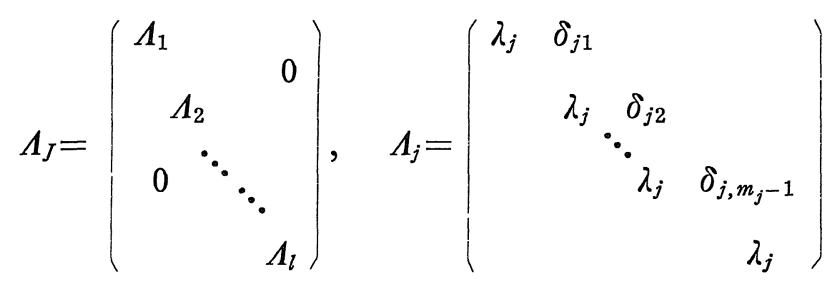

and each $\delta_{j k}$ satisfies the following inequality

$$
\left|\delta_{j k}\right| \leqq \delta
$$

Denoting the diagonal matrix $\operatorname{diag}\left(\lambda_{1}, \ldots, \lambda_{1}, \lambda_{2}, \ldots, \lambda_{2}, \ldots, \lambda_{l}, \ldots, \lambda_{l}\right)$ by $\Lambda_{D}$, we have

$$
\left\|\Lambda_{J}-\Lambda_{D}\right\| \leqq \delta
$$

Now, putting

$$
z(x, u)=S w(x, u)
$$

and substituting this in (7.13), we have the equation for $w$

$$
w\left(x+1, \pi u^{\Lambda}\right)=S^{-1} c(x, u)+S^{-1} B(x, u) S w+S^{-1} \psi(x, u, S w) .
$$

For simplicity, we put

$$
\begin{gathered}
\mathscr{C}(x, u)=S^{-1} c(x, u), \\
\mathscr{B}(x, u)=S^{-1} B(x, u) S, \\
\Psi(x, u, w)=S^{-1} \psi(x, u, S w) .
\end{gathered}
$$

Then (7.19) becomes

$$
w\left(x+1, \pi u^{\Lambda}\right)=\mathscr{C}(x, u)+\mathscr{B}(x, u) w+\Psi(x, u, w) .
$$


We may choose $R^{*}$ sufficiently large and $r^{*}(<1)$ sufficiently small so that the following conditions (A.I), (A.II) and (A.III) may be satisfied. (A.I) The components of $\mathscr{C}(x, u)$ are analytic in $X \times U$ where

$$
X:|x|>R^{*}, \quad U:\|u\|<r^{*},
$$

and the inequality

$$
\|\mathscr{C}(x, u)\| \leqq L_{1}\left\{|x|^{-(N+1)}+\|u\|^{N+1}\right\} \quad\left(L_{1}=\left\|S^{-1}\right\| L\right)
$$

holds in $X \times U$.

(A.II) The inequality

$$
\left\|\mathscr{B}(x, u)-\Lambda_{D}\right\| \leqq \delta+B\left\{|x|^{-1}+\|u\|\right\}
$$

holds in $X \times U$.

(A.III) The components of $\Psi(x, u, w)$ are analytic in $X \times U \times W$ such as

$$
X:|x|>R^{*}, \quad U:\|u\|<r^{*}, \quad W:\|w\|<r^{*},
$$

and the inequality

$$
\|\Psi(x, u, w)\| \leqq K_{1}\|w\|^{2} \quad\left(K_{1}=K\left\|S^{-1}\right\|\|S\|^{2}\right)
$$

holds in $X \times U \times W$.

8. Existence theorem. In this section, we shall prove that the system of the functional difference equation (7.20) has an analytic solution such that

$$
\|w(x, u)\| \leqq M\left\{|x|^{-(N+1)}+\|u\|^{N+1}\right\}
$$

in a certain region $\Gamma \times U_{0}$ such as

$$
\begin{array}{ll}
\Gamma:|x|>\rho_{1}, & |\arg x|<\alpha, \\
U_{0}:\|u\|<\rho_{2} & (<1)
\end{array}
$$

where $M$ and $\alpha$ are arbitrary but fixed constants such that $M>L$ $/\left(\lambda_{0}-1-\delta\right)$ and $0<\alpha<\frac{\pi}{2}$, and $\rho_{1}$ and $\rho_{2}$ are constants to be determined in the course of the proof. We shall employ the Fixed-Point Theorem to 
prove this.

Let $F$ be a family of vector-valued functions $w(x, u)$ whose elements are analytic and satisfy the inequality (8.1) for $(x, u)$ in the region $\Gamma \times U_{0}$.

Now, rewriting the equation (7.20), we have

$$
w(x, u)=\Lambda_{D}^{-1}\left\{w\left(x+1, \pi u^{\Lambda}\right)-\mathscr{C}(x, u)-\left(\mathscr{B}(x, u)-\Lambda_{D}\right) w-\Psi(x, u, w)\right\}
$$

where $\Lambda_{D}$ is the diagonal matrix $\operatorname{diag}\left(\lambda_{1}, \ldots, \lambda_{1}, \lambda_{2}, \ldots, \lambda_{2}, \ldots \ldots, \lambda_{l}, \ldots, \lambda_{l}\right)$.

Consider the mapping $T: w(x, u) \rightarrow \bar{w}(x, u)$ defined by

$$
\begin{aligned}
\bar{w}(x, u)= & T(w(x, u)) \\
= & \Lambda_{D}^{-1}\left\{w\left(x+1, \pi u^{A}\right)-\mathscr{C}(x, u)\right. \\
& \left.-\left(\mathscr{B}(x, u)-\Lambda_{D}\right) w-\Psi(x, u, w)\right\} .
\end{aligned}
$$

The most important fact to be proved is that if $w(x, u) \in F$ then also $\bar{w}(x, u) \in F$. We shall prove this only, since the rests may be proved similarly as in Part I.

We shall notice that if $(x, u) \in \Gamma \times U_{0}$ then $\left(x+1, \pi u^{\Lambda}\right) \in \Gamma \times U_{0}$, i.e., if $w(x, u) \in F$, then also $w\left(x+1, \pi u^{\Lambda}\right) \in F$. On the other hand, by (A.I), (A.II) and (A.III) the inequalities

$$
\begin{aligned}
& \|\mathscr{C}(x, u)\| \leqq L_{1}\left\{|x|^{-(N+1)}+\|u\|^{N+1}\right\} \\
& \left\|\mathscr{B}(x, u)-\Lambda_{D}\right\| \leqq \delta+B\left\{|x|^{-1}+\|u\|\right\}
\end{aligned}
$$

hold in the region $X \times U$ and the inequality

$$
\|\Psi(x, u, w)\| \leqq K_{1}\|w\|^{2}
$$

holds in the region $X \times U \times W$. By these facts we may get the inequality

$$
\begin{aligned}
\|\bar{w}(x, u)\| \leqq & \left\|\Lambda_{D}^{-1}\right\|\left[\left(M+L_{1}\right)\left(|x|^{-(N+1)}+\|u\|^{N+1}\right)\right. \\
& +\left(\delta+B\left(|x|^{-1}+\|u\|\right)\right) M\left(|x|^{-(N+1)}+\|u\|^{N+1}\right) \\
& \left.+K_{1} M^{2}\left(|x|^{-(N+1)}+\|u\|^{N+1}\right)^{2}\right] .
\end{aligned}
$$

By $\left\|\Lambda_{D}^{-1}\right\|=1 / \lambda_{0}$, the inequality 


$$
\begin{aligned}
\|\bar{w}(x, u)\| \leqq & \frac{1}{\lambda_{0}}\left[1+\frac{L_{1}}{M}+\delta+B\left(|x|^{-1}+\|u \mid\|\right)\right. \\
& \left.+K_{1} M\left(|x|^{-(N+1)}+\|u\|^{N+1}\right)\right] M\left(|x|^{-(N+1)}+\|u\|^{N+1}\right)
\end{aligned}
$$

holds in the region $X \times U$.

Let us choose $M$ so that $M>L_{1} /\left(\lambda_{0}-1-\delta\right)$, and let us take $\rho_{1}\left(>R^{*}\right)$ sufficiently large and $\rho_{2}\left(<r^{*}\right)$ sufficiently small. Then we have

$$
M=L /\left\{\lambda_{0}-1-\delta-B\left(|x|^{-1}+\|u\|\right)-K_{1} M\left(\|x\|^{-(N+1)}+\|u\|^{N+1}\right)\right\}
$$

in the region $\Gamma \times U_{0}$ which is contained in $X \times U$. Hence we get the inequality

$$
\frac{1}{\lambda_{0}}\left\{1+\frac{L_{1}}{M}+\delta+B\left(|x|^{-1}+\|u\|\right)+K_{1} M\left(|x|^{-(N+1)}+\|u\|^{N+1}\right)\right\} \leqq 1
$$

in $\Gamma \times U_{0}$. Therefore by (8.4), we have

$$
\|\bar{w}(x, u)\| \leqq M\left\{|x|^{-(N+1)}+\|u\|^{N+1}\right\}
$$

for any $w(x, u) \in F$. Hence the mapped function $\bar{w}(x, u)$ which is clearly analytic in $\Gamma \times U_{0}$ belongs also to $F$. Thus we get the following

Theorem 4. Consider the system of functional difference equations

$$
w\left(x+1, \pi u^{\Lambda}\right)=\mathscr{C}(x, u)+\mathscr{B}(x, u) w(x, u)+\Psi(x, u, w(x, u))
$$

where $\mathscr{C}(x, u), \mathscr{B}(x, u)$ and $\Psi(x, u, w)$ satisfy the conditions (A.I), (A.II) and (A.III) in $\S 7$. Equation (7.20) then has an analytic solution such that

$$
\|w(x, u)\| \leqq M\left\{|x|^{-(N+1)}+\|u\|^{N+1}\right\}
$$

in a certain region $\Gamma \times U_{0}=\left\{x|| x\left|>\rho_{1},\right| \arg x \mid<\alpha\right\} \times\left\{u \mid\|u\|<\rho_{2}<1\right\}$, where $M$ and $\alpha$ are arbitrary but fixed constants such that $M>L_{1} /\left(\lambda_{0}-1-\delta\right)$ and $0<\alpha<\frac{\pi}{2}$, and $\rho_{1}, \rho_{2}$ are suitable constants.

9. Uniqueness theorem. In this section, we shall consider the uniqueness of the solution of the functional difference equation 


$$
w\left(x+1, \pi u^{\Lambda}\right)=\mathscr{C}(x, u)+\mathscr{B}(x, u) w(x, u)+\Psi(x, u, w(x, u))
$$

under the conditions (A.I), (A.II) and (A.III) in $\S 7$. We shall prove that if (7.20) has an analytic solution satisfying $\|w(x, u)\| \leqq M\left\{|x|^{-(N+1)}+\|u\|^{N+1}\right\}$ in the region $\Gamma \times U_{0}$, then such a solution is unique.

Let us assume that there exist two solutions $w_{1}(x, u)$ and $w_{2}(x, u)$. Put

$$
v(x, u)=w_{2}(x, u)-w_{1}(x, u),
$$

then $v$ satisfies the following equation

$$
v\left(x+1, \pi u^{\Lambda}\right)=\mathscr{B}(x, u) v+\Psi\left(x, u, w_{1}+v\right)-\Psi(x, u, v) .
$$

Expanding the right-hand side in power of $v$, we can write the above equation in the form

$$
v\left(x+1, \pi u^{\Lambda}\right)=A\left(x, u, w_{1}\right) v+\Psi_{1}\left(x, u, w_{1}, v\right),
$$

where $\Psi_{1}$ denotes the part containing terms of degrees higher than one in $v$.

Let us choose constants $\bar{R}_{1}\left(\geqq \max \left(\rho_{1}, R^{*}\right)\right)$ sufficiently large, and $\bar{r}_{1}\left(=\min \left(\rho_{2}, r^{*}\right)\right)$ sufficiently small, and consider the regions $D_{1}, U_{1}, W_{1}$ and $V_{1}$ defined by

$$
\begin{aligned}
& D_{1}:|x| \geqq \bar{R}_{1}, \quad|\arg x|<\alpha, \\
& U_{1}:\|u\| \leqq \bar{r}_{1}, \\
& W_{1}:\left\|w_{1}\right\| \leqq M\left(\bar{R}_{1}^{-(N+1)}+\bar{r}_{1}^{N+1}\right) \\
& V_{1}:\|v\| \leqq 2 M\left(\bar{R}_{1}^{-(N+1)}+\bar{r}_{1}^{N+1}\right) .
\end{aligned}
$$

Then we may assume that $A\left(x, u, w_{1}\right)$ is analytic in the region $D_{1} \times U_{1} \times W_{1}$ and the equality

$$
A(\infty, 0,0)=\Lambda_{J}
$$

holds, where $\Lambda_{J}$ is the Jordan's canonical form (7.16) of $\Lambda=\left(\lambda_{i j}\right)$. On the other hand, $\Psi_{1}\left(x, u, w_{1}, v\right)$ is analytic in the region $D_{1} \times U_{1} \times W_{1} \times V_{1}$ and the inequality 


$$
\left\|\psi_{1}\left(x, u, w_{1}, v\right)\right\| \leqq K_{2}\|v\|^{2}
$$

holds in $D_{1} \times U_{1} \times W_{1} \times V_{1}$, where $K_{2}$ is a constant suitably chosen.

Set $H\left(x, u, w_{1}\right)=A\left(x, u, w_{1}\right)-\Lambda_{J}$, then (9.3) becomes

$$
v\left(x+1, \pi u^{\Lambda}\right)=\left(\Lambda_{J}+H\left(x, u, w_{1}\right)\right) v+\Psi_{1}\left(x, u, w_{1}, v\right) .
$$

As is easily seen, the inequality

$$
\left\|\Lambda_{J} v\right\| \geqq\left(\lambda_{0}-\delta\right)\|v\|
$$

holds for any $v$, where $\lambda_{0}=\min \left|\lambda_{j}\right|$ and $\delta$ is a constant satisfying

$$
\lambda_{0}-1>\delta>0 \text {. }
$$

Now let us choose an arbitrary but fixed positive constant $\delta_{1}$ such that

$$
\lambda_{0}-1-\delta>\delta_{1}>0
$$

Then, choosing suitably the constants $\bar{R}_{2}, \bar{r}_{2}, \overline{\bar{r}}_{2}$ and $\tilde{r}_{2}$ the inequality

$$
\left\|H\left(x, u, w_{1}\right) v+\Psi_{1}\left(x, u, w_{1}, v\right)\right\| \leqq \delta_{1}\|v\|
$$

holds in the region $V=D_{2} \times U_{2} \times W_{2} \times V_{2}$ such as

$$
\begin{aligned}
& D_{2}:|x| \geqq \bar{R}_{2} \quad\left(\geqq \bar{R}_{1}\right), \quad|\arg x|<\alpha, \\
& U_{2}:\|u\| \leqq \bar{r}_{2} \quad\left(\leqq \bar{r}_{1}\right), \\
& W_{2}:|| w_{1} \| \leqq M\left(\bar{R}_{2}^{-(N+1)}+\bar{r}_{2}^{N+1}\right) \equiv \overline{\bar{r}}_{2} \\
& V_{2}:\|v\| \leqq 2 M\left(\bar{R}_{2}^{-(N+1)}+\bar{r}_{2}^{N+1}\right) \equiv \tilde{r}_{2} .
\end{aligned}
$$

By (9.5), (9.6) and (9.8), we have

$$
\begin{aligned}
& \left\|v\left(x+1, \pi u^{\Lambda}\right)\right\| \\
& \quad=\left\|\Lambda_{J} v+H\left(x, u, w_{1}\right) v+\Psi_{1}\left(x, u, w_{1}, v\right)\right\| \\
& \quad \geqq\left\|\Lambda_{J} v\right\|-\left\|H\left(x, u, w_{1}\right) v+\Psi_{1}\left(x, u, w_{1}, v\right)\right\| \\
& \quad \geqq\left(\lambda_{0}-\delta\right)\|v\|-\delta_{1}\|v\| \\
& \quad=\left(\lambda_{0}-\delta-\delta_{1}\right)\|v\|
\end{aligned}
$$

in the region $V$. Putting $\rho=\lambda_{0}-\delta-\delta_{1}$ we get $\rho>1$ by (9.7). Then the inequality 


$$
\left\|v\left(x+1, \pi u^{\Lambda}\right)\right\| \geqq \rho\|v\|
$$

holds in the region $V$. Since $v$ is bounded in the region $D_{2} \times U_{2}$, putting $S=\sup _{D_{2} \times U_{2}}\|v(x, u)\|_{\text {! we }}$ have $S \geqq \rho S$ where $\rho>1$. Hence $S=0$, i.e., $w_{1}(x, u)=w_{2}(x, u)$. The solution being analytic $\Gamma \times U$, such a solution is unique in $\Gamma \times U$. Thus we get the following

Theorem 5. Under the conditions of Theorem 4 the solution stated in it is unique.

The existence of an analytic asymptotic solution can be proved in a similar manner as in Part I by using Theorem 4, Theorem 5 and the inequality $\|z\| \leqq\|S\|\|w\|$ in (7.18). Thus we have

Theorem 6. Under the conditions of Theorem 4 there exists an analytic solution of (7.3) for which we have the asymptotic expansion

$$
y_{i}(x, u) \sim u_{i}\left(1+\sum_{|k| \geqq 1} p_{k_{0} k_{1} \ldots k_{m}}^{(i)} x^{-k_{0}} u_{1}^{k_{1}} u_{2}^{k_{2}} \cdots u_{m}^{k_{m}}\right)
$$

in the region $\Gamma \times U$.

\section{References}

[1] Harris Jr., W. A. and Y. Sibuya, Asymptotic solutions of systems of nonlinear difference equations, Arch. Rational Mech. Anal. 15 (1964), 377-395.

[2] Harris Jr., W. A. and Y. Sibuya, General solution of nonlinear difference equations, Trans. Amer. Math. Soc. 115 (1965), 62-75.

[ 3 ] Harris Jr., W. A., On a theorem of S. Tanaka, Publ. Res. Inst. Math. Sci. Kyoto Univ. Ser. A, 2 (1966), 1-4.

[4] Horn, J., Zur Theorie der nichtlinearen Differenzen-gleichungen, J. Reine Angew. Math. 141 (1912), 182-216.

[5] Hukuhara, M., Sur les points singuliers d'une équation différentielle ordinaire du premier ordre, I, Mem. Fac. Eng. Kyushu Imp. Univ. 8 (1937), 203-247.

[6] Hukuhara, M., Renzokuna Kansu no Zoku to Shazo, (Japanese), Mem. Fac. Sci. Kyushu Univ. Ser. A, 5 (1950), 61-63.

[7] Tanaka, S., On asymptotic solutions of non-linear difference equations, I, Mem. Fac. Sci. Kyushu Univ. Ser. A, 7 (1953), 107-127.

[8] Tanaka, S., On asymptotic solutions of non-linear difference equations, II, Mem. Fac. Sci. Kyushu Univ. Ser. A, 10 (1956), 45-83.

[9] Tanaka, S., On asymptotic solutions of non-linear difference equations, III, Mem. Fac. Sci. Kyushu Univ. Ser. A, 11 (1957), 167-184.

[10] Trjitzinsky, W. J., Non-linear difference equations, Compositio Math. 5 (1937-1938), $1-60$. 